\title{
INVESTIGATING GEOSPARQL REQUIREMENTS FOR PARTICIPATORY URBAN PLANNING
}

\author{
E. Mohammadi a, *, A. J.S. Hunter ${ }^{\mathrm{a}}$ \\ ${ }^{a}$ Geomatics Department, Schulich School of Engineering, University of Calgary, Calgary, T2N 1N4, Alberta, CANADA \\ - (emohamma, ahunter)@ucalgary.ca
}

Commission VI, WG VI/4

KEY WORDS: GeoSPARQL, PGIS, Urban Planning, Spatial Reasoning, Urban Patterns, Spatial Relations

\begin{abstract}
:
We propose that participatory GIS (PGIS) activities including participatory urban planning can be made more efficient and effective if spatial reasoning rules are integrated with PGIS tools to simplify engagement for public contributors. Spatial reasoning is used to describe relationships between spatial entities. These relationships can be evaluated quantitatively or qualitatively using geometrical algorithms, ontological relations, and topological methods. Semantic web services utilize tools and methods that can facilitate spatial reasoning. GeoSPARQL, introduced by OGC, is a spatial reasoning standard used to make declarations about entities (graphical contributions) that take the form of a subject-predicate-object triple or statement. GeoSPARQL uses three basic methods to infer topological relationships between spatial entities, including: OGC's simple feature topology, RCC8, and the DE-9IM model. While these methods are comprehensive in their ability to define topological relationships between spatial entities, they are often inadequate for defining complex relationships that exist in the spatial realm. Particularly relationships between urban entities, such as those between a bus route, the collection of associated bus stops and their overall surroundings as an urban planning pattern. In this paper we investigate common qualitative spatial reasoning methods as a preliminary step to enhancing the capabilities of GeoSPARQL in an online participatory GIS framework in which reasoning is used to validate plans based on standard patterns that can be found in an efficient/effective urban environment.
\end{abstract}

\section{INTRODUCTION}

Participatory GIS (PGIS) applications provide opportunities for residents of communities to share their ideas, concerns and opinions about how their environment should be planned and developed. In order to fully explore the opinions of participants, data collection methodologies should verify the compatibility of the ideas and diagrams contributed against standard patterns so as to provide feedback about the implications of a particular contribution. To this end, PGIS would be enhanced by equipping them with spatial reasoning logic to describe relationships between entities encountered during a mapping and planning activity.

The overall purpose of the research that this paper is based on, is to contribute to Participatory GIS (PGIS) by contributing new tools that allow sketching as a medium for collecting local planning ideas. The tools that have been developed in this research are intended to support freehand sketching, a mapping/drafting/design approach that from a conceptual perspective should not limit participants' ability to participate in a PGIS activity. Having a good understanding of current sketch recognition algorithms and the design of an appropriate interface is essential for this purpose. Sketching is one aspect of this research (however, this paper is not discussing this aspect); accurate and reliable interpretation of sketches from the planning domain is another. This necessitates a comprehensive knowledge of spatial relationships encountered within the planning domain, supported by an ontology of planning objects.
This paper is about the basic study of the relationships between urban entities and how these relationships can be modelled to facilitate the reasoning. Reasoning is based on some predefined rules in urban planning and the objective of this paper is to define these rules and simplify them in order to design intelligent (free-sketch based) tools for public use that transforms average people's (i.e. people with no urban planning expertise) sketches of space into meaningful urban entities. For this purpose GeoSPARQL and its models are studied as the major geospatial ontological modelling language.

OGC's GeoSPARQL is a prominent standard for geospatial reasoning. The topological properties of features can be queried with GeoSPARQL, and can be expressed using three distinct vocabularies: OGC's Simple Features topological relations; Egenhofer's DE-9IM model; and RCC8 (Battle \& Kolas, 2011).

Simple features topological relations include equals, disjoint, intersects, touches, within, contains, overlaps and crosses. The DE-9IM model uses the concept of interior $\left({ }^{\circ}\right)$, boundary $(\partial)$, and exterior (') of features to describe their topological relationships. The matrix given in figure 1 presents the topological relationships that can be modeled by DE-9IM. Application of DE-9IM to two geometries will produce a pattern that is associated with a topological relationship.

\footnotetext{
* Corresponding author. Ehsan Mohammadi (emohamma@ucalgary.ca)
} 


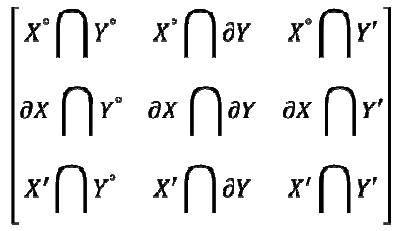

Figure 1. DE-9IM Matrix

RCC8 uses the concept of connection between regions to describe relationships (Cohn, Gooday, Bennett, \& Gotts, 1995; Cohn \& Hazarika, 2001; Cohn \& Renz, 2008; Kontchakov, Pratt-Hartmann, \& Zakharyaschev, 2014). The connection relation is reflexive and symmetric. Figure 2 illustrates possible RCC8 concepts for two regions. Using RCC8, the following topologies can be described: disjoint (DC), meet (EC), overlap (PO), inside (NTTP), contains (NTTPi), covers (TTPi), covered by (TPP), and equal (EQ).
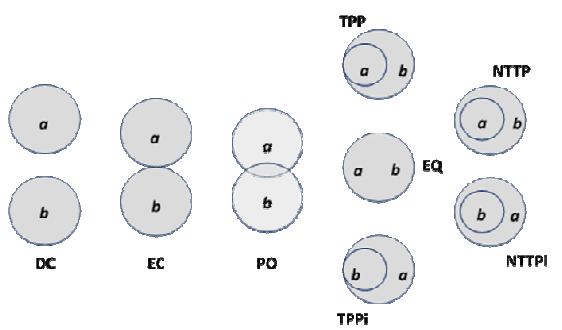

Figure 2. RCC8 Topological Model

Although these models can represent most planning topological relations, they lack some essential relations required in many urban planning applications. Missing relations limits the usefulness of PGIS applications when attempts are made to interpret the sketches made by practitioners. For instance, when practitioners design new road networks, intersection requirements, determined by road classification, will affect the arrangement of adjacent buildings. Describing this type of relationship is beyond the capabilities of the aforementioned models.

\section{QUALITATIVE SPATIAL REPRESENTATION AND REASONING}

There are many different aspects to space (e.g. discrete space vs. continuous space and absolute space vs. relative space) and therefore to its representation and reasoning. Qualitative Spatial Reasoning (QSR) is an active research area covering representation and reasoning in the spatial domain. Works by Cohn (Cohn \& Hazarika, 2001; Cohn \& Renz, 2008) augment spatial representation and reasoning with the concepts of: ontology, mereology, mereotopology, mereogeometry (the study and mathematics of part-whole relationships), spatial relations, direction and orientation, shape, distance and size, spatial vagueness and uncertainty. Freksa (1992) and Zimmermann \& Freksa (1996) discuss qualitative spatial reasoning based on directional orientation information. In their work, 15 qualitatively different relations are defined between a point and a vector (directed line segment). Figure 3 illustrates these relations. These relationships can be perceived by human mind by terms of being left/right, ahead/behind, aligned with line/normal to line at end points and so on.

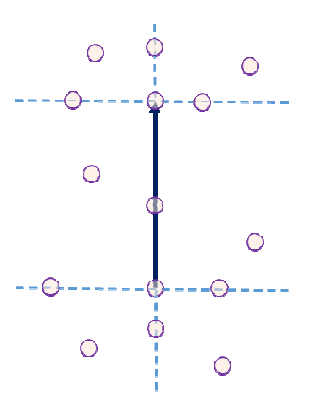

Figure 3. 15 Qualitatively Different Relations of a Point and a Vector

Moratz, Renz \& Wolter (2000) present an approach for dealing with intrinsic orientation information by specifying qualitative relations between oriented line segments. They identify a set of 24 atomic relations between two distinct oriented lines, based on the relation of right/left/on between each line and start/end points of another line (e.g. if a street is completely to the right of a highway) Frank $(1992,1996)$ addresses spatial reasoning using cardinal directions as a qualitative method emphasizing differences between human understanding of directional relations and their quantitative values. All these reasoning calculi facilitate the process of modelling relationships between urban spatial entities.

\section{URBAN ENTITIES RELATIONSHIPS}

"A pattern language" by Christopher Alexander (Alexander et al., 1977) describes more than 250 patterns found in urban planning. The first 94 patterns are relevant to the public realm, roads, parks, public centres, etc. Development of the public realm often engages local residents during early planning, offering them an opportunity to contribute to the (re)design and (re)development of their community. Studying these patterns gives insight into how urban areas can be configured, and how various features should be arranged as intertwining components to shape efficient and lively public spaces. These patterns can be used as a guide when assessing different scenarios during an urban planning activity, especially one in which the public are participating. However, we admit that patterns should not be restricted solely to the ones contained in Alexander et al's (1977) book alone, and that more contemporary patterns can be identified through the study of current urban develops and urban design theory. For this work, we use Alexander et al's (1977) work as a benchmark.

Urban patterns reveal how constituent entities in an urban environment can be arranged, and the type of relationships that exist between them. From a spatial reasoning point of view these arrangements and relationships can be modelled using geometric, topological, and semantic relationships, or a combination of them.

All the entities in an urban planning environment can be modelled as objects. These objects have shape (geometry) and position, among other spatial and non-spatial properties. Considering the combination of objects in an urban area reveals the type of relationships they have to each other. Based on Alexander et al's (1977) work, we extracted the major relationships expected to be found in a vibrant urban area. In order to assist contributors during an engagement process, these 
relationships should be built into an intelligent informationgathering tool. The intent is to enhance participation by offering direction to participants that reflects good planning design practice.

\subsection{Ontological Relationships}

Here we describe the classification of urban entities and their relationships used in this investigation.

All physical entities found in an urban environment are spatial objects that occupy some extent. They can be modelled using simple or complex polygons. However, from a spatial modelling and/or cartographic perspective, some objects can be modelled as linear or point objects at different scales. Considering a range of scales from small to large we assume that objects can be represented by a single, simple spatial entity such as a point, line or polygon, or as complex spatial entities consisting of multiple points, multiple lines, or multiple polygons, or combinations of (multi) points, (multi) lines and (multi) polygons.

Along with the spatial primitives used to describe objects, the hierarchy of objects and their classes and sub-classes (e.g. different classes of roads or land use) are also an ontological concern. Being part of another entity (whole-part relation) is considered to be an ontological relationship, which is also common in urban environment (e.g. a bus stop is part of a route in a transportation network).

\subsection{Topological Relationships}

As discussed, simple topology, DE-9IM and RCC8 are common topology models. However, they mostly represent relationships between regions, and are appropriate for simple topological relationships (such as adjacency or containment), as opposed to complex relationships (e.g. house cluster patterns and their relationships with different classes of roads) commonly found in urban environments.

\subsection{Geometrical Relationships}

Geometric functions of urban entities are also important. Each entity may contain constraints that can be described by its geometry. The area of a community, the length of a road, the maximum distance between transit stops in a local transportation system are all examples of geometric attributes or rules. Geometric functions or relationships can be classified as:

1. Unary: these functions are applied to single entities. Determining concavity vs. convexity, simplicity vs. complexity, area, perimeter, length, centroid, and position are examples of unary geometric functions.

2. Binary: these functions work on two entities. Distance between two points, maximum and minimum distance of a point from a polygon, and orientation of two line segments with regard to each other are some binary geometric relationships.

3. $\mathrm{N}$-ary: $\mathrm{N}$-ary functions are applied to more than two entities. The geometric distance between stops in a transportation network, the location of storm water catchbasins along a road network (at sag points, at the [uphill] start of curves at an intersections, etc.) considers many entities together.
Distance and direction (orientation) are two main functions that play crucial roles in binary and $\mathrm{N}$-ary relationships. Despite distance being a simple concept it can be calculated many ways in geographic space. Direct Euclidean distance, network distance, Manhattan distance, travel distance and geodesic distance are all examples illustrating its complexity. Adding a vertical dimension to space allows one to add additional distance metrics such as horizontal, vertical and slope distance. Operationally, distance can also be measure in length, time to traverse between two points, or the cost of traversing a route.

There are also additional considerations when estimating distance between points, lines and polygons. The distance between point, line and polygon entities may also be determined in a number of ways, which adds to the complexity of the problem. The Hausdorff distance or the maximum distance between two entities is commonly used when defining geometrical relationships based on distance.

Direction or orientation is also significant when defining spatial relationships. As with distance, direction or orientation also has many meanings based on the frame of reference being used. It could be based on an individual entity (front, back, left, right, behind and next are examples of this ego-centric frame of reference). A frame of reference can be relative to other entities (perpendicular to, aligned with and parallel to are examples of directional relations between two entities). Finally, a frame of reference can be external to all entities (North, South, East, and West are all directions that are independent of specific entities and are absolute concepts rather than relative ones).

\subsection{Patterns}

Urban entities are rarely considered on their own. They are components of a larger integrated system. In this regard, urban entities usually have meaningful relationships with other entities that create distinguishable patterns. Modelling these types of relationships is important, and requires that combinations of relationships be used to express patterns reliably.

How the complexity of relationships between urban entities can be simplified by mentioned relationships can be more illustrated by an example showing the context sensitivity of the applied rules: Let's assume a zone around the periphery of a parcel that you cannot place a building within. This is a type of distance metric between two polygons at a typical design scale. The building has to be within the parcel, the edge of the building closest to the road must be at some distance from the edge of the parcel closest to the road. Orientation is defined by the relationship between the road and the parcel. This allows the identification of side and rear yard building restrictions. From an implementation perspective we would use a buffer, but we still need to know where the road is relative to the parcel as the side yard restrictions are generally less than the front and rear yards (at least in typical residential zones).

In the next section some of the specific types of relationships that can be observed between urban entities are discussed. These relationships can be regarded as complex relationships that can be decomposed into a set or combinations of simple relationships. 


\section{SPECIFIC URBAN RELATIONSHIPS}

These specific urban relationships form the backbone of patterns that can be found in a functioning urban area. Some of them may seem to be simple relationships, but to model them requires more than one operation. The following describe some of the specific relationships that exist between urban entities:

1. Workplaces should be within 20 to 30 minutes travel time from housing. This relationship defines a maximum distance (Hausdorff distance) between two entities or two clusters of entities: living areas and working areas.

2. A town should be a mosaic of communities. This relationship means that all communities should be covered or contained by a town area; the intersection of communities is only allowed at their boundaries (no overlaps); and the union of all communities should be equal to town area.

3. A town should be divided into local transport areas. This relationship is a little bit more complicated since local transport areas can intersect each other, and the union of all transportation catchments should cover the whole town.

4. Houses areas (and common or park lands) should create clusters. Creating a cluster is more a statistical function combined with a distance function. However, some entities (including housing and common lands) should be arranged in meaningful clusters in a city.

5. Common or parklands should be surrounded by houses and work places. Being around some other entity requires a disjoint relation as well as being covered by a specific buffer from the entity.

6. Major roads should be approximately parallel at a specific distance within a town or city to reduce traffic congestion, and distribute traffic volume over the city. Parallel lines at a certain distance apart is the type of relationship here. However, being parallel is more a fuzzy concept than a crisp one. The transportation network (pedestrian, cycling, private, and public transportation) forms a hierarchy, where essentially the distance between constituent parts of the network is a function of scale. The pedestrian network might consist of a $100 \mathrm{~m}$ grid, which is overlaid with a $400 \mathrm{~m}$ grid for cycling, etc.

7. Shopping streets should be perpendicular to major roads. Perpendicular is a fuzzy concept between two linear entities.

8. Sidewalks should run along minor roads. To be along is a relationship between two linear entities and requires orientation and distance operations used together.

9. Shopping centres should have parking lots behind them. Being behind another entity is an orientation operation, and requires a frame of reference based on the major entity.

10. Local transport areas should be surrounded by a ring road, or a country town should be surrounded by countryside. Surrounding, or being surrounded by another entity is a complex relationship, since it can be a partial surrounding or a complete surrounding. When an entity surrounds another entity, they are disjoint, but the surrounding entity's bounding box contains the surrounded entity, and they are at least a specific distance from each other.

11. A boundary separates two communities. A boundary can be considered any entity that separates two other entities. A boundary can be a road, green infrastructure (a pathway along a stream or bush area), a river, an industrial area, or other similar feature.

12. An industrial area can be part of a boundary. Being part of another entity is an ontological relationship, however geometric and topological relationships must also be applied.

13. Activity nodes should be distributed evenly in a community. This relationship is a statistical operation with a number of internal parameters, including distance, population, and area.

The urban relationships mentioned are only a few of the specific relationships between urban entities. Modelling all relationships within a spatial reasoning platform will require comprehensive definition of them.

\section{CONCLUSIONS}

This paper is the first attempt to recognize different types of relationships that exist between urban entities and shed some light on requirements of a comprehensive reasoning engine in order to extend the capabilities of GeoSPARQL to make it more suitable for an intelligent PGIS tool. Defining the specific relationships requires combination of geometric, topological and semantic relationships and should be investigated thoroughly based on defined patterns. Investigation of spatial relations of urban entities is the fundamental step in designing a participatory platform that accepts free-hand sketching by contributors as input, and interprets them to determine if they are valid urban entities. This is the next step in this research.

The provision of sketch tools will enable users to create markups and annotate existing development plans, create and publish their own models as alternative plans, and modify proposed plans. The intelligence that can be built using GEOSPARQL, DE-9IM, RCC8, etc., allows the complexity and language of planning design rules to be hidden from the user, simplifying the interaction neccesary to participate. This is important for promoting engagement of community members in local planning issues as it promotes 'handing over the stick' (Chambers, 2007).

\section{REFERENCES}

Alexander, C., Ishikawa, S., Silverstein, M., Jacobson, M., Fiksdahl-King, I., \& Angel, S. (1977). A pattern language: Towns, buildings, construction. New York: Oxford University Press.

Battle, R., \& Kolas, D. (2011). Geosparql: enabling a geospatial semantic web. Semantic Web J, 3(4), 355-370.

Chambers, R. (2007). Who Counts? The Quiet Revolution of Participation and Numbers. Brighton, UK: Institute of Development Studies.

Cohn, A. G., Gooday, J. M., Bennett, B., \& Gotts, N. M. (1995). A logical approach to representing and 
reasoning about space. Artificial Intelligence Review, 9(4-5), 255-259.

Cohn, A. G., \& Hazarika, S. M. (2001). Qualitative spatial representation and reasoning: An overview. Fundamenta Informaticae, 46(1), 1-29.

Cohn, A. G., \& Renz, J. (2008). Qualitative spatial representation and reasoning. Handbook of Knowledge Representation, 3, 551-596.

Frank, A. U. (1992). Qualitative spatial reasoning about distances and directions in geographic space. Journal of Visual Languages \& Computing, 3(4), 343-371.

Frank, A. U. (1996). Qualitative spatial reasoning: Cardinal directions as an example. International Journal of Geographical Information Science, 10(3), 269-290.

Freksa, C. (1992). Using orientation information for qualitative spatial reasoning. Springer. Retrieved from http://link.springer.com/chapter/10.1007/3-54055966-3_10

Kontchakov, R., Pratt-Hartmann, I., \& Zakharyaschev, M. (2014). Spatial reasoning with RCC8 and connectedness constraints in Euclidean spaces. Artificial Intelligence, 217, 43-75.

Moratz, R., Renz, J., \& Wolter, D. (2000). Qualitative spatial reasoning about line segments. In ECAI (pp. 234238). Citeseer. Retrieved from http://citeseerx.ist.psu.edu/viewdoc/download?doi=10 .1.1.84.1377\&rep=rep $1 \&$ type $=$ pdf

Zimmermann, K., \& Freksa, C. (1996). Qualitative spatial reasoning using orientation, distance, and path knowledge. Applied Intelligence, 6(1), 49-58.

Revised April 2015 